\title{
A case of invasive cytomegalovirus duodenitis in an immunosuppressed patient 15 months after renal transplantation
}

\author{
N. Kazanji · F. Davila $\cdot$ P. Manickam • Y. Wang · \\ L. Bossory
}

Received: 25 October 2014 / Accepted: 18 December 2014 / Published online: 13 January 2015

(C) Springer-Verlag Berlin Heidelberg 2015

\begin{abstract}
Cytomegalovirus (CMV) remains one of the most important infections in kidney transplantation. Only a handful of images have been reported in the literature thus far. We present classic pathologic and gross images of CMV duodenitis in an immunosuppressed patient more than one year post-renal transplantation.
\end{abstract}

Keywords Cytomegalovirus - CMV duodenitis - Renal transplant · Viremia $\cdot$ Immunocompromised $\cdot$ Duodenitis

N. Kazanji $(\bowtie) \cdot$ F. Davila

Department of Internal Medicine, Oakland University William

Beaumont School of Medicine, Beaumont Health System,

3601 W 13 Mile Rd, Royal Oak, MI 48073, USA

e-mail: noora.kazanji@beaumont.edu

F. Davila

e-mail: Francisco.Davila@beaumont.edu

\section{P. Manickam}

Department of Gastroenterology, Oakland University William Beaumont School of Medicine, Beaumont Health System, 3601 W 13 Mile Rd, Royal Oak, MI 48073, USA

e-mail: palaniappan.manickam@beaumont.edu

\section{Y. Wang}

Department of Pathology, Oakland University William Beaumont School of Medicine, Beaumont Health System, 3601 W 13 Mile Rd, Royal Oak, MI 48073, USA

e-mail: Yingchun.Wang@beaumont.edu

\section{Bossory}

Oakland University, Oakland University William Beaumont School of Medicine, 2200 N Squirrel Rd, Rochester, MI 48309,

USA

e-mail: lbossory@oakland.edu
Case

A 65-year-old male presented with 10 days of abdominal discomfort, vomiting, and diarrhea. He underwent renal transplantation 15 months prior due to diabetic and hypertensive nephropathy with donor-positive and recipientnegative cytomegalovirus (CMV) IgG. He was maintained on mycophenolate, prednisone, and tacrolimus. Nucleic acid amplification detected $97,400 \mathrm{IU} / \mathrm{mL}$ of CMV consistent with viremia. He developed severe sepsis and was placed on valgancyclovir as only antimicrobial. On day 5 , he developed bloody stools requiring transfusions and fluid resuscitation. His colonoscopy was normal. Esophagogastroduodenoscopy showed non-bleeding superficial duodenal ulcers (Fig. 1). Duodenal biopsy revealed cytomegaloviral inclusion bodies and positivity for p52 on immunohistochemistry (Fig. 2), indicating CMV duodenitis. The bleeding and diarrhea resolved after 5 and 30 days, respectively. His viral load became undetectable on day 30 of therapy.

Cytomegalovirus remains one of the most important infections in kidney transplantation. CMV duodenitis has been infrequently described, although recent studies found that CMV is activated easier in the upper rather than lower gastrointestinal tract [1, 2]. Images of CMV duodenitis have been seldom reported, mainly in patients with acquired immunodeficiency syndrome [3-5]. According to Sarkio et al. [2], duodenal inclusion bodies were detected only in $15 \%$ of cases, all of them within a year post-transplantation. Given the scarcity and variety of CMV duodenitis histopathology, it is crucial that additional images are identified. We present classic images of primary CMV duodenitis in a patient more than one year post-renal transplantation. This entity should be suspected in any immunocompromised patient with persistent gastrointestinal symptoms. 

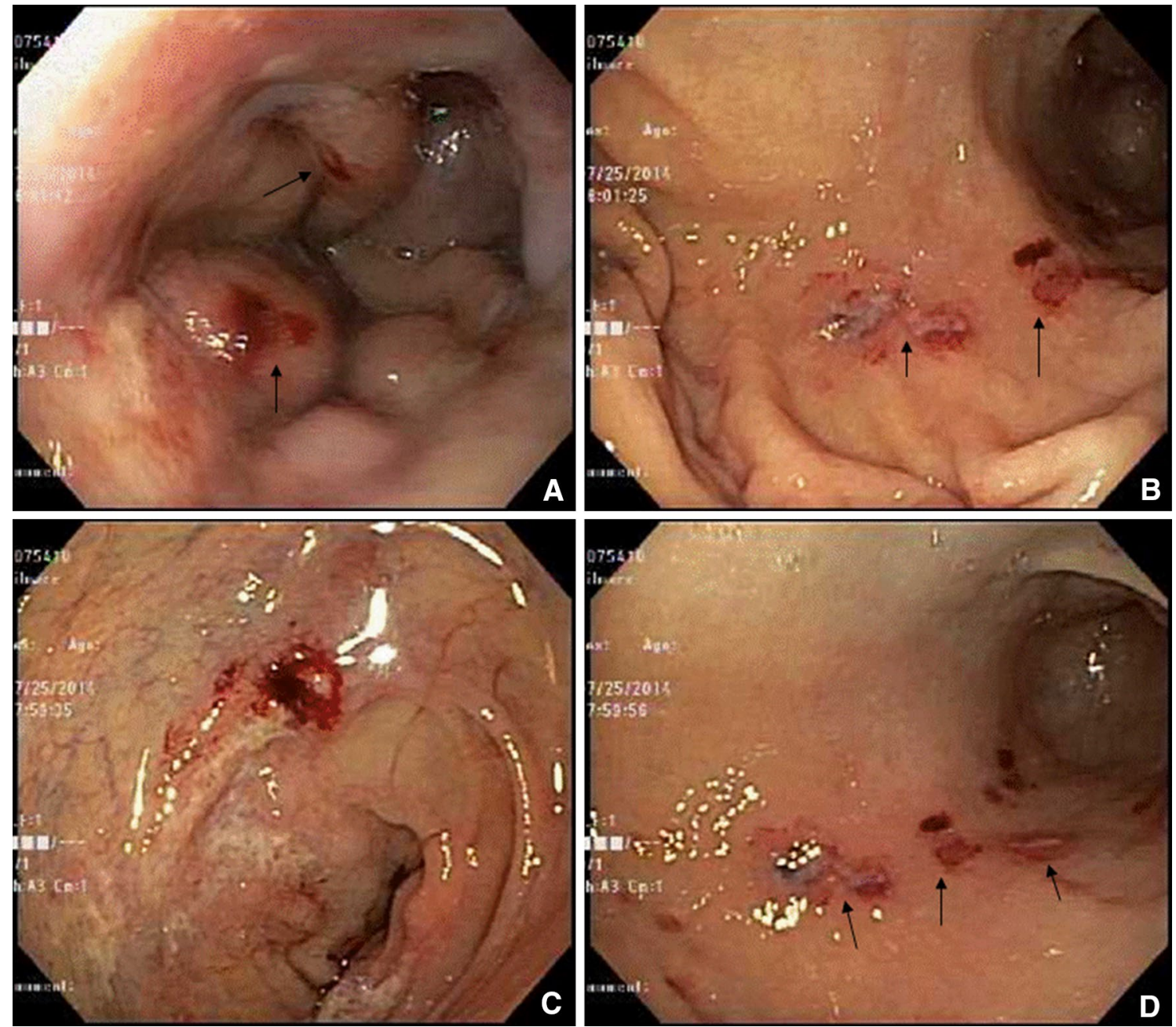

Fig. 1 Esophagogastroduodenoscopy photograph showing nonbleeding superficial duodenal ulcers and erosions (noted by arrows) duodenum (a), junction of first and second parts of the duodenum (b), duodenal bulb (c, d). Post-biopsy changes are shown in c. The largest lesion was $13 \mathrm{~mm}$ in dimension 


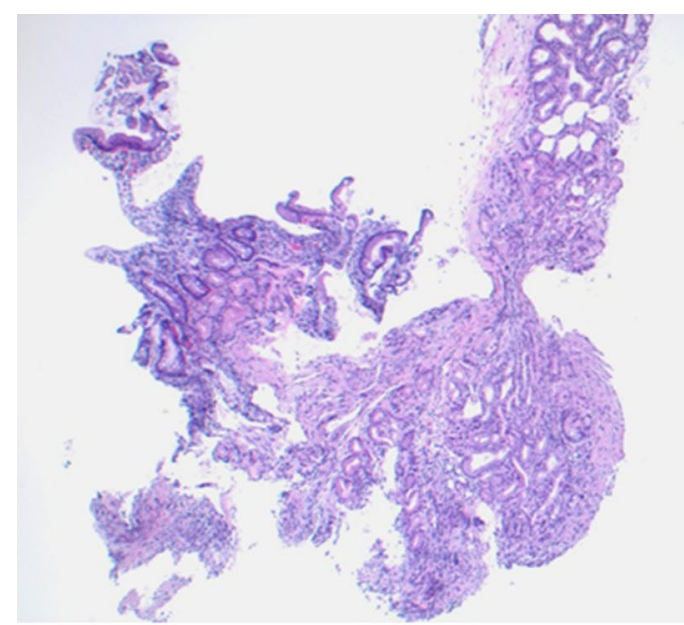

A
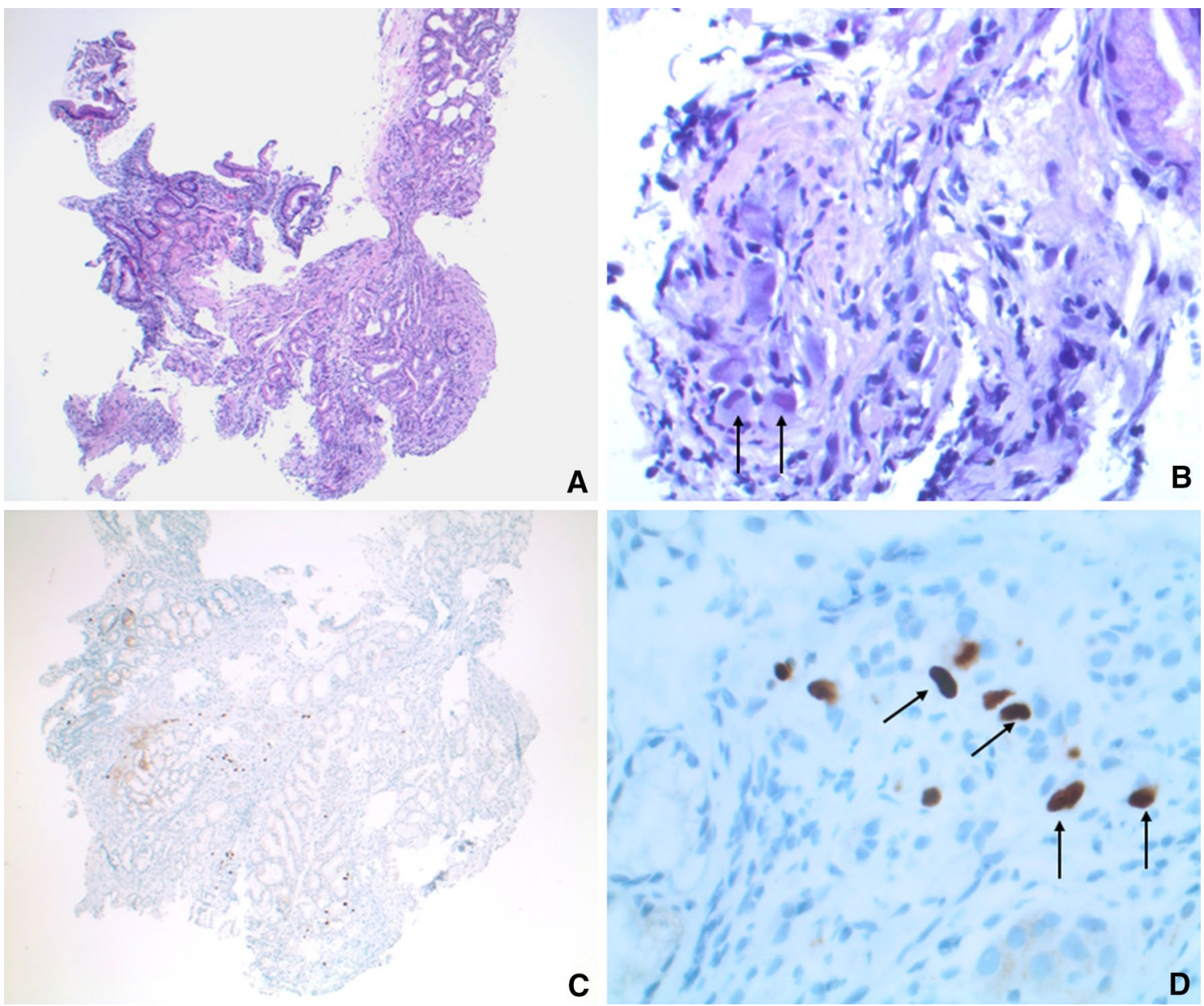

Fig. 2 Duodenal mucosa with mucosal erosion $($ a $\mathrm{HE} \times 40)$ and cytomegaloviral cytopathic change with inclusion bodies noted by arrows (b $\mathrm{HE} \times 400)$. Immunohistochemistry for CMV shows posi-

tivity, noted by arrows, of DNA-binding protein, p52 using isotype controls with IgG1, kappa of the $\mathrm{CCH} 2$ antibody and IgG2a, kappa of the DDG9 antibody. $($ c. $\times 40$ and $\mathbf{d} . \times 400)$

Conflict of interest On behalf of all authors, the corresponding author states that there is no conflict of interest.

\section{References}

1. Lempinen M, Halme L, Sarkio S, et al. CMV findings in the gastrointestinal tract in kidney transplantation patients, patients with end-stage kidney disease and immunocompetent patients. Nephrol Dial Transpl. 2009. doi:10.1093/ndt/gfp408.

2. Sarkio S, Halme L, Arola J. Gastroduodenal cytomegalovirus infection is common in kidney transplantation patients. Scand $\mathrm{J}$ Gastroenterol. 2005;40:508-14.

3. Kelesidis T, Tozzi S, Mittyet R, et al. Cytomegalovirus pseudotumor of the duodenum in a patient with AIDS: an unrecognized and potentially treatable clinical entity. Int $\mathrm{J}$ Infect Dis. 2010;14:e274-82.

4. Wilcox CM, Schwartz DA. Symptomatic CMV duodenitis. An important clinical problem in AIDS. J Clin Gastroenterol. 1992;14:293-7.

5. Falasca F, Maida P, Gaeta et al. A detection and quantification of EBV, HHV-6 and CMV DNA in the gastrointestinal tract of HIV-positive patients. Infection. 2014; 42:1033-7. doi: 10.1007/s15010-014 0690-5. Epub 2014 Oct 18. PubMed PMID: 25326253. 